\title{
BOUSSINESQ CONVECTION IN A GASEOUS SPHERICAL SHELL
}

\author{
L. Korre ${ }^{1}$, N. Brummell ${ }^{1}$ and P. Garaud ${ }^{1}$
}

\begin{abstract}
In this paper, we investigate the dynamics of convection in a spherical shell under the Boussinesq approximation but considering the compressibility which arises from a non zero adiabatic temperature gradient, a relevant quantity for gaseous objects such as stellar or planetary interiors. We find that depth-dependent superiadiabaticity, combined with the use of mixed boundary conditions (fixed flux/fixed temperature), gives rise to unexpected dynamics that were not previously reported.
\end{abstract}

\section{Introduction}

Convection is a very common physical process in astrophysical and geophysical fluid dynamics which has been extensively studied since it plays a fundamental role in the global dynamics of the Earth's oceans and atmosphere, as well as in stars and planets. A common method for studying convection using numerical simulation is to use the Boussinesq approximation in a chosen geometry.

In the vast majority of spherical Boussinesq studies of convection published to date, fixed temperature boundary conditions are considered, such that convection is driven by a temperature difference between the top and bottom boundary (see Gastine et al. 2015 and references therein). This setup is well known as RayleighBénard convection (RBC thereafter). Here, we modify the boundary conditions to use mixed boundary conditions such that we have fixed flux at the bottom and fixed temperature at the top. This setup is more realistic for planetary and stellar models than the use of Dirichlet conditions and, as we will demonstrate, gives rise to unexpected new phenomena.

In many RBC studies, the fluid is assumed to be incompressible in the traditional Boussinesq sense (Boussinesq 1903). What is less commonly known is that the Boussinesq approximation can also be applied to model the dynamics of

${ }^{1}$ Department of Applied Mathematics and Statistics, Jack Baskin School of Engineering, University of California Santa Cruz, 1156 High Street, Santa Cruz, CA 95064, USA 
weakly compressible gases in the Earth's atmosphere or the interiors of stars or giant planets (Spiegel \& Veronis 1960, SV hereafter). Assuming that the modeled region is much smaller than any of the intrinsic scaleheights of the problem and that the velocity of the fluid is much smaller than the speed of sound, SV showed that the momentum and mass conservation equations of motion reduce to those used in the standard Boussinesq equations. The only difference is the appearance of a new term in the thermal energy equation which captures the adiabatic heating or cooling of a parcel of fluid as it shrinks or expands to adapt to the local background pressure.

To the authors' knowledge, in all Boussinesq RBC in spherical shells studies to date, this added compressional heating/cooling term was neglected, making them more relevant to studies of convection in liquids (water, molten metals or magma) than in gases. In order to model planetary or stellar interiors, however, this term must be present. As we shall demonstrate, it then calls for the introduction of a new non-dimensional parameter which had not previously been discussed.

In Section 2, we introduce our spherical shell weakly compressible Boussinesq convection model and associated boundary conditions. In Section 3, we discuss our numerical results. In Section 4, we provide a summary of the basic ideas and results of this paper as well as some implications that these results might have.

\section{The model}

We consider a spherical shell located between an inner sphere of radius $r_{i}=0.7 R$ and an outer sphere of radius $r_{o}=R$. We impose fixed flux at the bottom and fixed temperature at the top boundary. The dimensional SV Boussinesq equations in this setup are:

$$
\begin{gathered}
\rho_{m}\left(\frac{\partial \boldsymbol{u}}{\partial t}+\boldsymbol{u} \cdot \nabla \boldsymbol{u}\right)=-\nabla p+\tilde{\rho} \boldsymbol{g}+\rho_{m} \nu \nabla^{2} \boldsymbol{u} \\
\nabla \cdot \boldsymbol{u}=0 \\
\frac{\partial T}{\partial t}-u_{r} \frac{d T_{\mathrm{ad}}}{d r}+\boldsymbol{u} \cdot \nabla T=\nabla(\kappa \nabla T),
\end{gathered}
$$

where $\boldsymbol{u}$ is the velocity, $p$ the pressure, $T$ the temperature, $\tilde{\rho}$ is the density perturbation away from the mean density of the shell $\rho_{m}, \nu$ is the viscosity, $\kappa$ is the thermal diffusivity, $\alpha$ is the thermal expansion coefficient and $d T_{\mathrm{ad}} / d r=-g / c_{p}$ (where $g$ is the gravity and $c_{p}$ the specific heat at constant pressure) is the adiabatic temperature gradient. In the absence of fluid motion and when the system is in a steady state, we have that $T=T_{\text {rad }}(r)$ where $T_{\text {rad }}$ satisfies

$$
\kappa \nabla^{2} T_{\mathrm{rad}}(r)=0 \Rightarrow \kappa r^{2} \frac{d T_{\mathrm{rad}}}{d r}=\mathrm{const}=\kappa r_{o}^{2} \frac{d T_{o}}{d r}, \quad \text { with }\left|\frac{d T_{o}}{d r}=\frac{d T_{\mathrm{rad}}}{d r}\right|_{r=r_{o}} .
$$

We then let $T=T_{\text {rad }}+\Theta$, where $\Theta$ are the temperature perturbations. The linearized equation of state then becomes $\tilde{\rho} / \rho_{m}=-\alpha \Theta$. We non-dimensionalize 
these equations using the lengthscale $[l]=r_{o}$, the timescale $[t]=r_{o}^{2} / \nu$, the velocity scale $[u]=\nu / r_{o}$ and the temperature scale $[T]=\left|d T_{o} / d r-d T_{\mathrm{ad}} / d r\right| \cdot r_{o}$. Then, the nondimensional Boussinesq equations (where we have dropped the hats) are:

$$
\begin{gathered}
\nabla \cdot \boldsymbol{u}=0 \\
\frac{\partial \boldsymbol{u}}{\partial t}+\boldsymbol{u} \cdot \nabla \boldsymbol{u}=-\nabla p+\frac{\mathrm{Ra}_{\mathrm{o}}}{\operatorname{Pr}} \Theta \boldsymbol{e}_{r}+\nabla^{2} \boldsymbol{u} \\
\frac{\partial \Theta}{\partial t}+\beta(r) u_{r}+\boldsymbol{u} \cdot \nabla \Theta=\frac{\nabla^{2} \Theta}{\operatorname{Pr}}
\end{gathered}
$$

where the Prandtl and Rayleigh numbers are defined, respectively, as $\operatorname{Pr}=\nu / \kappa$ and $\mathrm{Ra}_{o}=\alpha g\left|\frac{d T_{o}}{d r}-\frac{d T_{\mathrm{ad}}}{d r}\right| r_{o}^{4} / \nu \kappa$ and where we introduce the function

$\beta(r)=\frac{\frac{d T_{\mathrm{rad}}}{d r}-\frac{d T_{\mathrm{ad}}}{d r}}{\left|\frac{d T_{o}}{d r}-\frac{d T_{\mathrm{ad}}}{d r}\right|}=\frac{\left(\frac{1}{r}\right)^{2} \frac{d T_{o}}{d r}-\frac{d T_{\mathrm{ad}}}{d r}}{\left|\frac{d T_{o}}{d r}-\frac{d T_{\mathrm{ad}}}{d r}\right|}=-\frac{\operatorname{Ra}(r)}{\mathrm{Ra}_{\mathrm{o}}}=-\frac{\frac{\alpha g\left|\frac{d T_{\mathrm{rad}}}{d r}-\frac{d T_{\mathrm{ad}}}{d r}\right| r_{o}^{4}}{\kappa \nu}}{\mathrm{Ra}_{o}}$.

Note that the $\mathrm{RBC}$ equations in a Cartesian setup can be recovered simply by taking the limit of a very thin shell $(r \rightarrow 1)$. In that case, $\beta(r) \approx$ const $\approx$ -1 , independently of $d T_{\mathrm{ad}} / d r$. In the spherical shell case on the other hand, the radiative temperature gradient is a function of radius, while the adiabatic temperature gradient is constant (assuming that $g$ and $c_{p}$ are constant). Hence $|\beta|$ increases inwards, as shown in Figure 1a.

The function $\beta$ can be rewritten more simply in terms of a single parameter $\chi$, defined as

$$
\chi=\left|\frac{\frac{d T_{o}}{d r}-\frac{d T_{\mathrm{ad}}}{d r}}{\frac{d T_{o}}{d r}}\right|, \quad \text { so } \quad \beta(r)=\frac{\left(1-\chi-(1 / r)^{2}\right)}{\chi} .
$$

When $d T_{\mathrm{ad}} / d r=0, \chi=1$ and effectively drops out leaving only the $\mathrm{Ra}_{o}$ and Pr numbers to dictate the dynamics of the system. We see, however, that when $d T_{\mathrm{ad}} / d r \neq 0$, the new parameter $\chi$ appears and can also influence the dynamics of convection, in addition to $\mathrm{Ra}_{o}$ and $\mathrm{Pr}$.

\section{Numerical results}

We now analyze the results of a series of numerical simulations which we have run using the PARODY-JA code (Aubert et al. 2008). We employ stress-free boundary conditions for the velocity field and we have fixed $\operatorname{Ra}_{o}=10^{7}$ and $\operatorname{Pr}=0.1$. We then vary $\chi$ in order to establish what effect this parameter has on the solutions. 

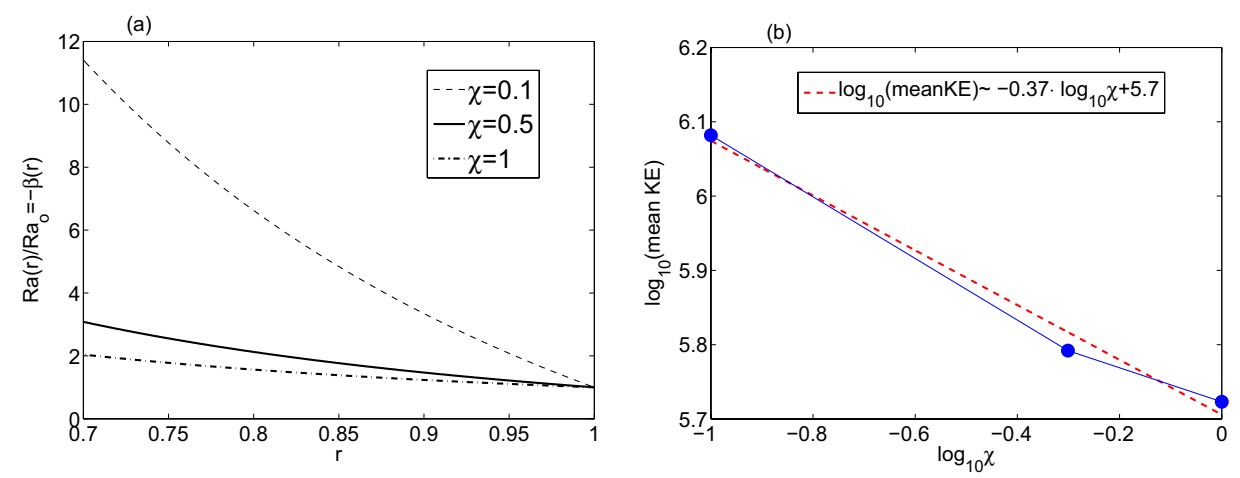

Fig. 1. a) The $\mathrm{Ra}(r) / \mathrm{Ra}_{o}$ function for three different values of $\chi$. b) Mean kinetic energy value for $\chi=0.1, \chi=0.5$ and $\chi=1$ for $\mathrm{Ra}_{o}=10^{7}$ and $\operatorname{Pr}=0.1$. The dashed line is the fitted line to the points.
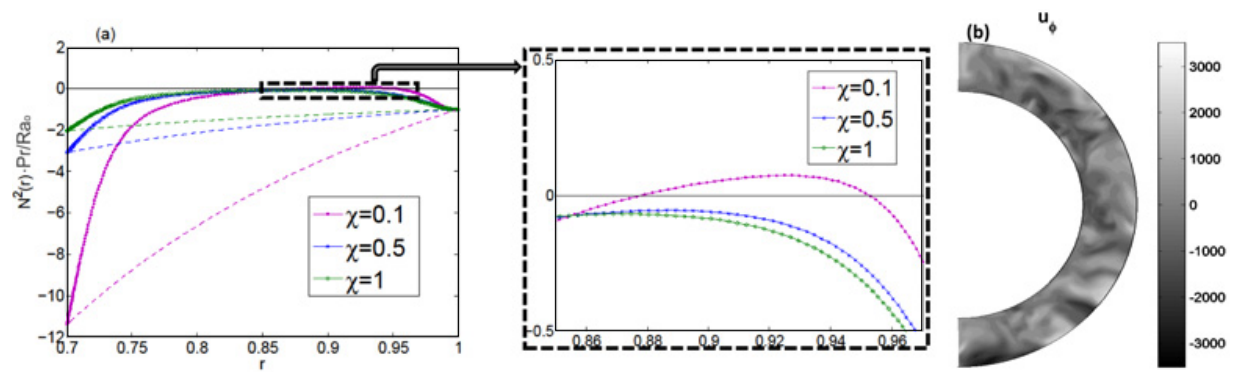

Fig. 2. a) Total $N^{2}(r)$ profile (solid lines) compared with that of the radiative background (dashed lines) for different values of $\chi$. b) Meridional slice snapshot of $u_{\phi}$ for the simulation with $\chi=0.1$.

In Figure 1b, we plot the mean kinetic energy (KE) in the simulation against $\chi$, for the three runs available. We observe that the mean kinetic energy increases substantially with decreasing $\chi$. We interpret this finding by the fact that the Rayleigh number increases more dramatically with depth for small values of $\chi$ so that convection is driven, overall, more vigorously.

To illustrate the effect of convection on the mean temperature profile, we present the non-dimensional buoyancy frequency, $N^{2}(r)=(\beta(r)+d \Theta / d r) \cdot\left(\mathrm{Ra}_{\mathrm{o}} / \mathrm{Pr}\right)$, compared with the one based on the background radiative temperature gradient (Fig. 2a).

We see that convection efficiently mixes the interior as expected, bringing $N^{2}$ close to zero. However, for smaller $\chi$ we see the unexpected emergence of a slightly stable region $\left(N^{2}>0\right)$ just below the upper boundary layer. The convection is still efficient there as can be seen from a meridional slice snapshot of the velocity in the $\phi$-direction for the $\chi=0.1$ case. The convective eddies are turbulent 
throughout the whole region (Fig. 2b). This unexpected result points to strong non-local effects, where convective eddies simply coast through this stable region while being driven on either sides of it.

\section{Discussion}

We have studied Boussinesq convection in a spherical shell and have observed that some unexpected dynamics emerge, most notably the appearance of a region that superficially appears linearly stable to convection and yet the convection seems apparently unaffected, suggesting highly non-local motions. It is important then to ascertain under which conditions such dynamics arise, and to elucidate what are the essential ingredients that lead to such unexpected results.

Although typical Boussinesq RBC assumes fixed temperature boundary conditions, here we choose to used mixed boundary conditions due to their greater relevance for astrophysical objects. These, along with the geometry of the spherical shell, and the inclusion of a non-zero adiabatic temperature gradient via the function $\beta(r)$ which varies most when $\chi$ is small, enforce a higher degree of asymmetry on the flows than usual. The resultant convection is then very asymmetrical with the top boundary dynamics being very different from the ones at the bottom boundary. However, convection is still efficient everywhere, which indicates that it is a very non-local process.

The predominant up-down asymmetry of the convective eddies is likely to have a strong effect on penetrative convection, a topic close to the heart of Jean-Paul Zahn (e.g. Zahn 1991). In follow-up publications, we shall present a more comprehensive understanding of the various effects described in this proceedings, and will then proceed to analyze their quantitative impact on penetrative convection, with application to the solar tachocline.

The authors acknowledge financial support from NASA NNX14AG08G. The numerical simulations were performed on Hyades supercomputer at UCSC purchased using an NSF MRI grant.

\section{References}

Aubert, J., Aurnou, J., \& Wicht, J., 2008, Geophys. J. Int., 172, 945

Boussinesq J. 1903, Paris: Gauthier-Villars, 2, 172

Gastine T., Wicht J., \& Aurnou J., 2015, J. Fluid Mech., 778, 721

Korre, L., Brummell, N., \& Garaud, P., 2017, Phys. Rev. E, 96, 033104

Spiegel, E.A., \& Veronis, G., 1960, ApJ, 131, 442

Zahn, J.-P., 1991, A\&A, 252, 179 
Astro Fluid 2016

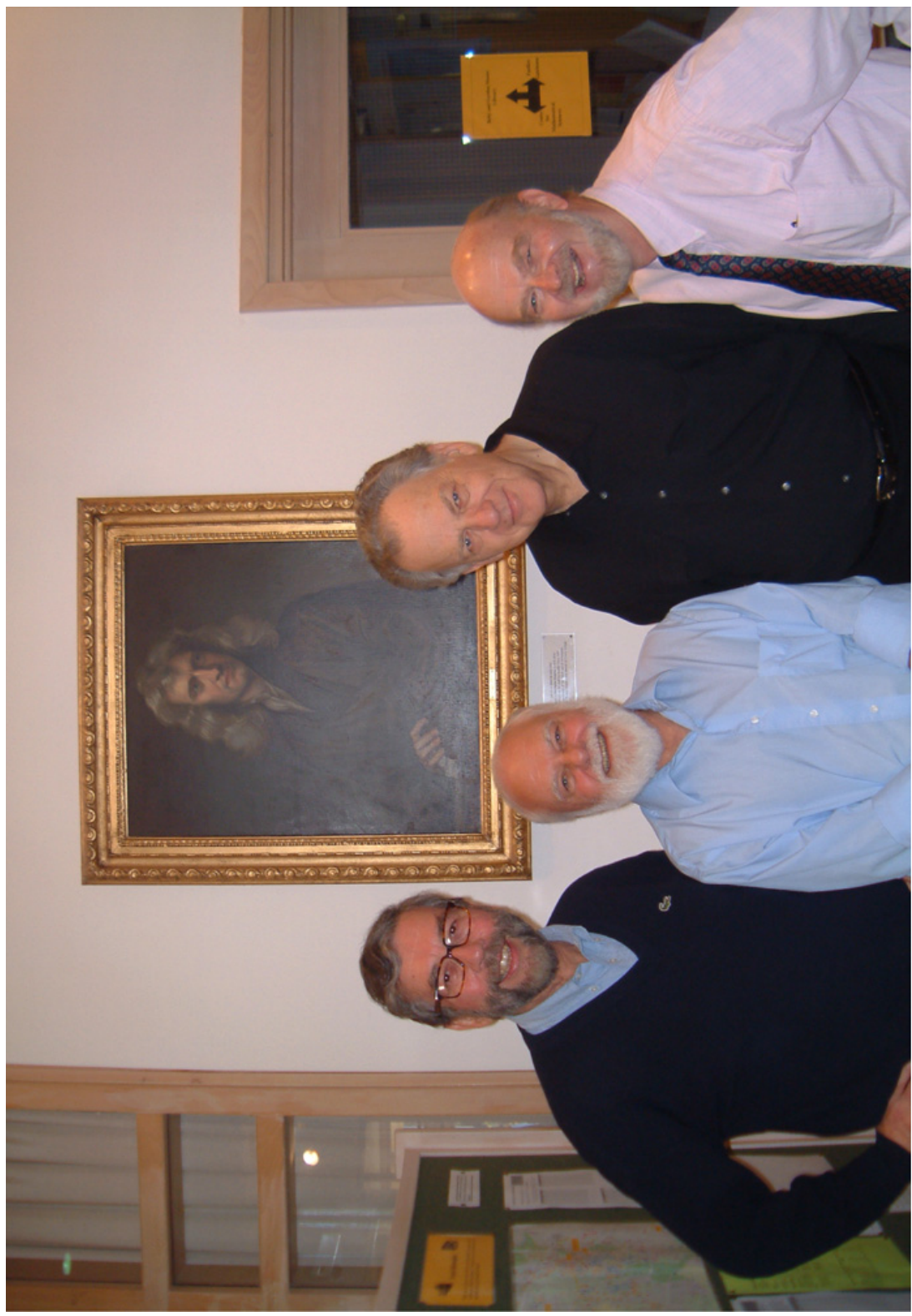

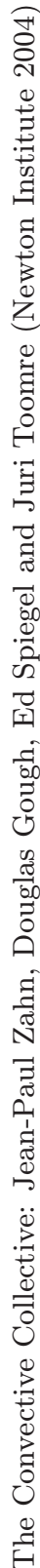




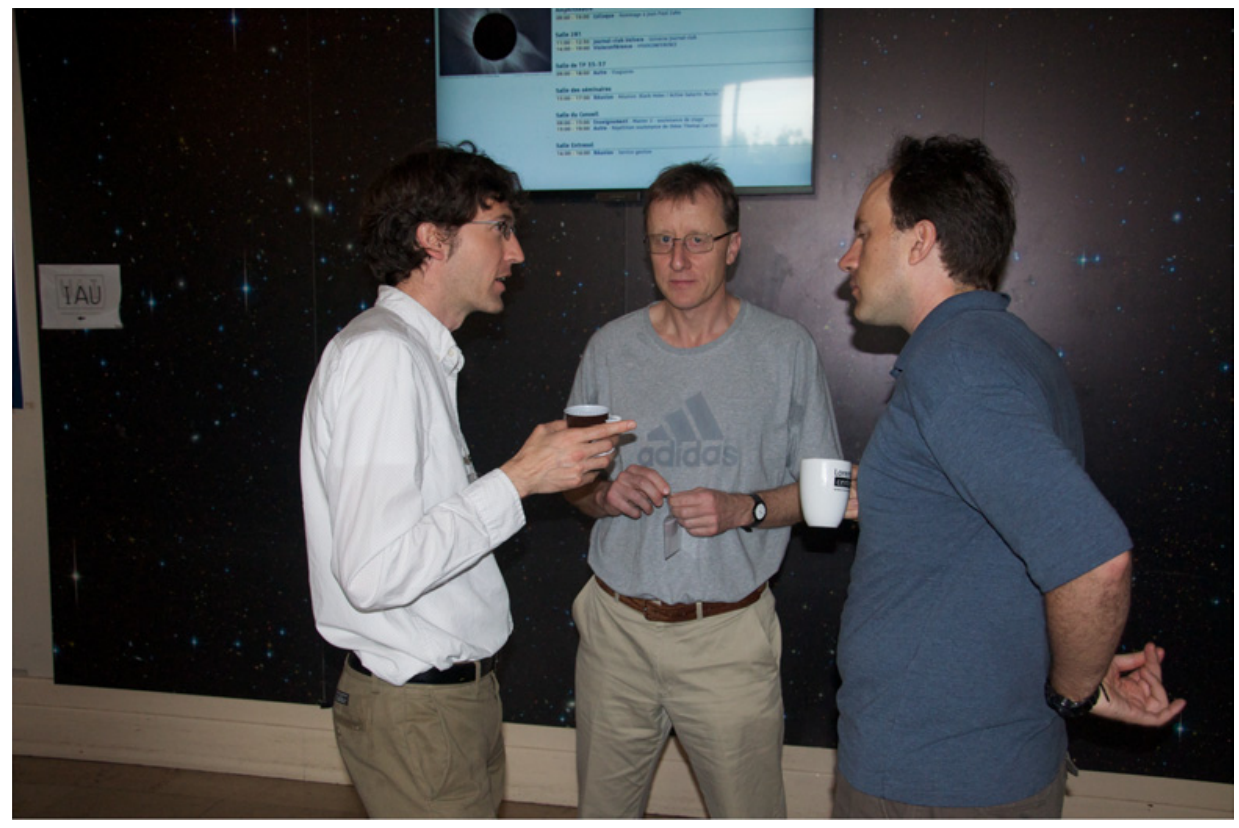

Matt Browning and David Hughes

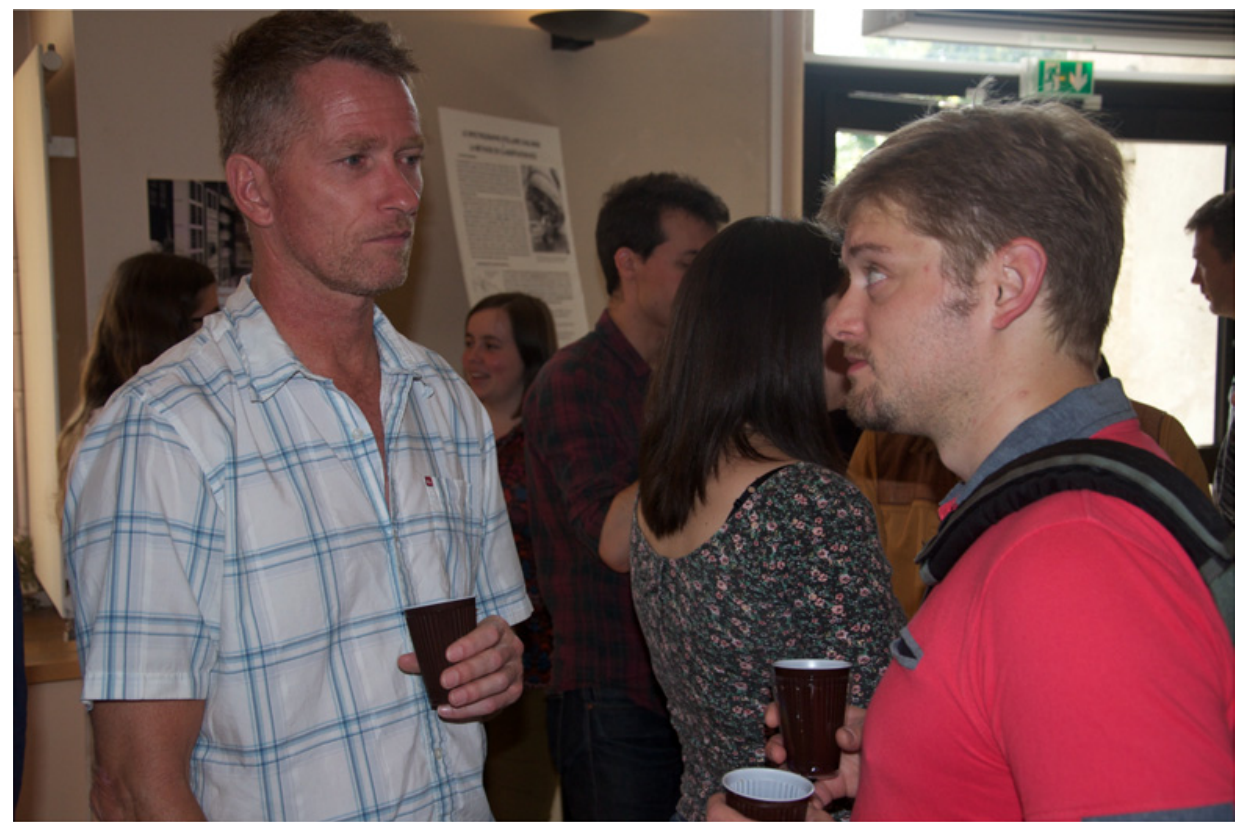

Nicholas Brummell and Kyle Augustson 


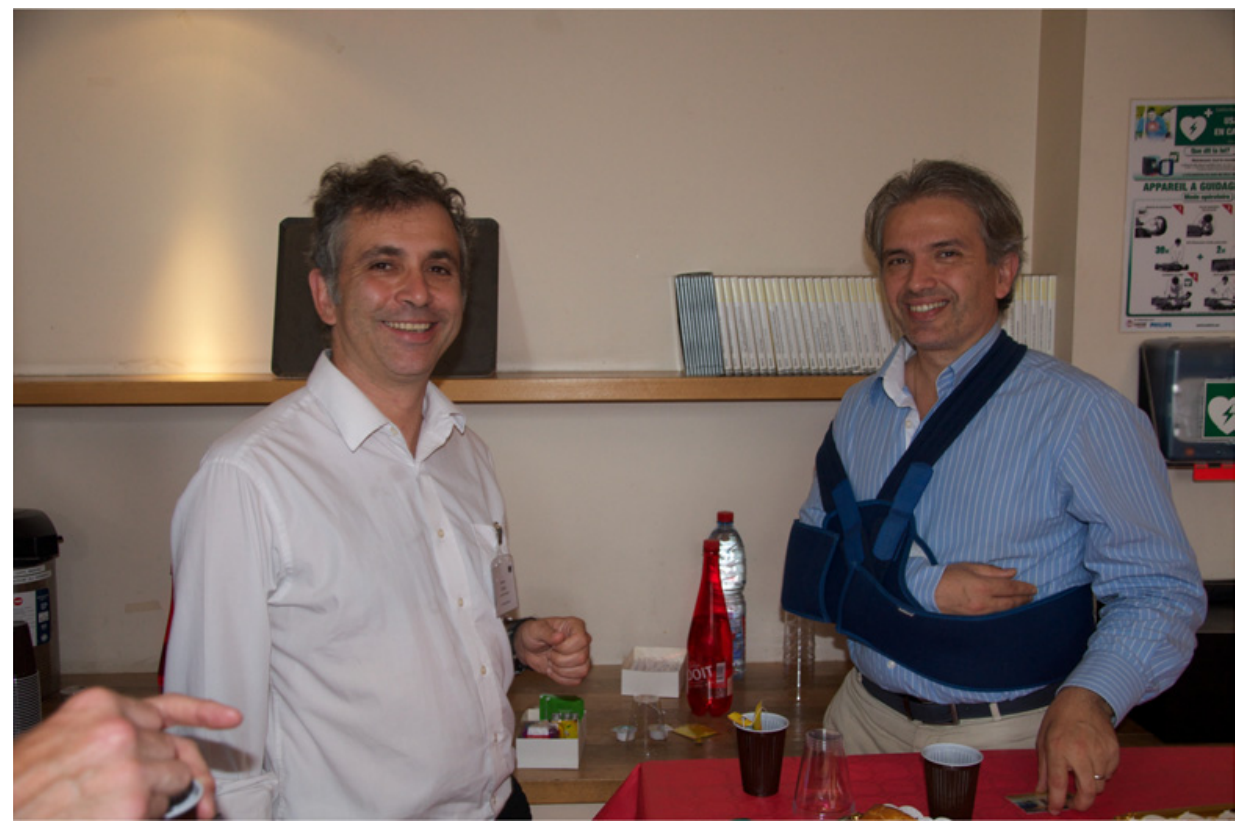

Steven Tobias and Allan Sacha Brun

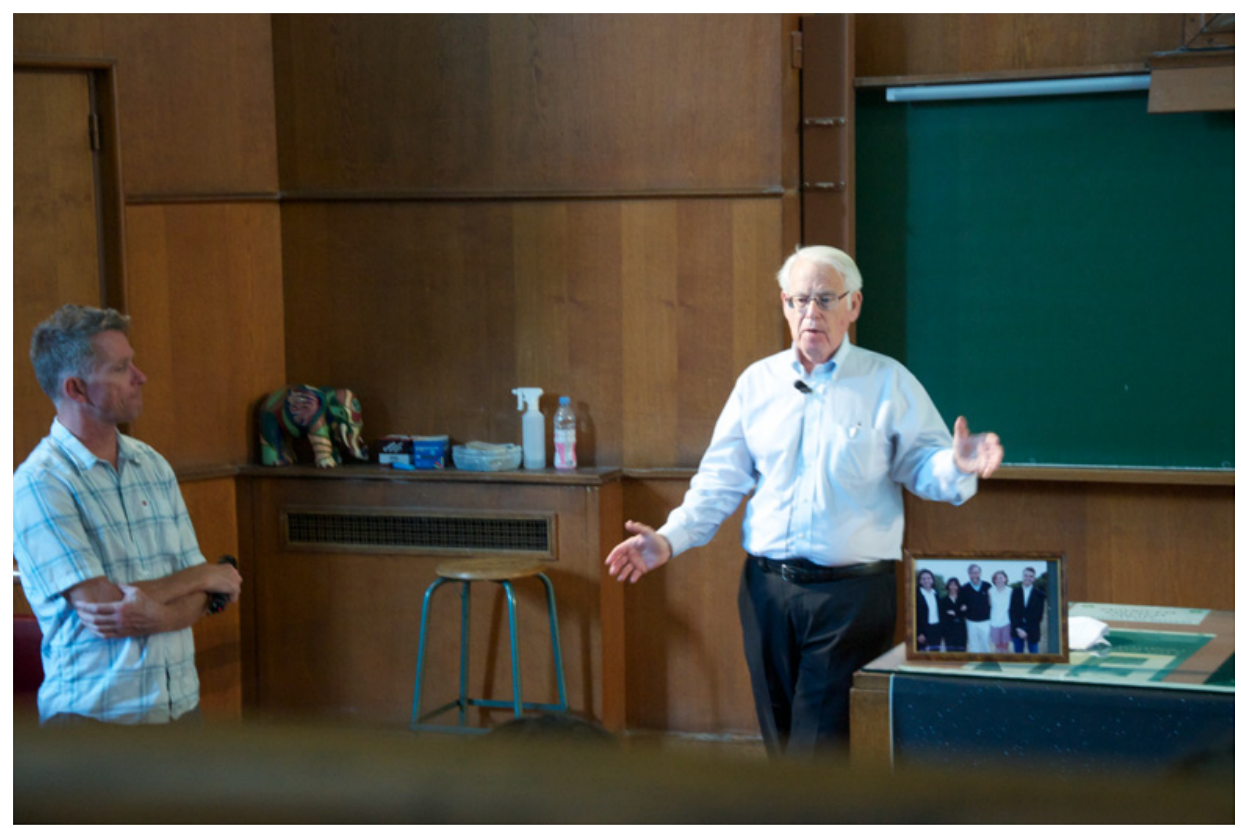

Nicholas Brummell and Alan Title 


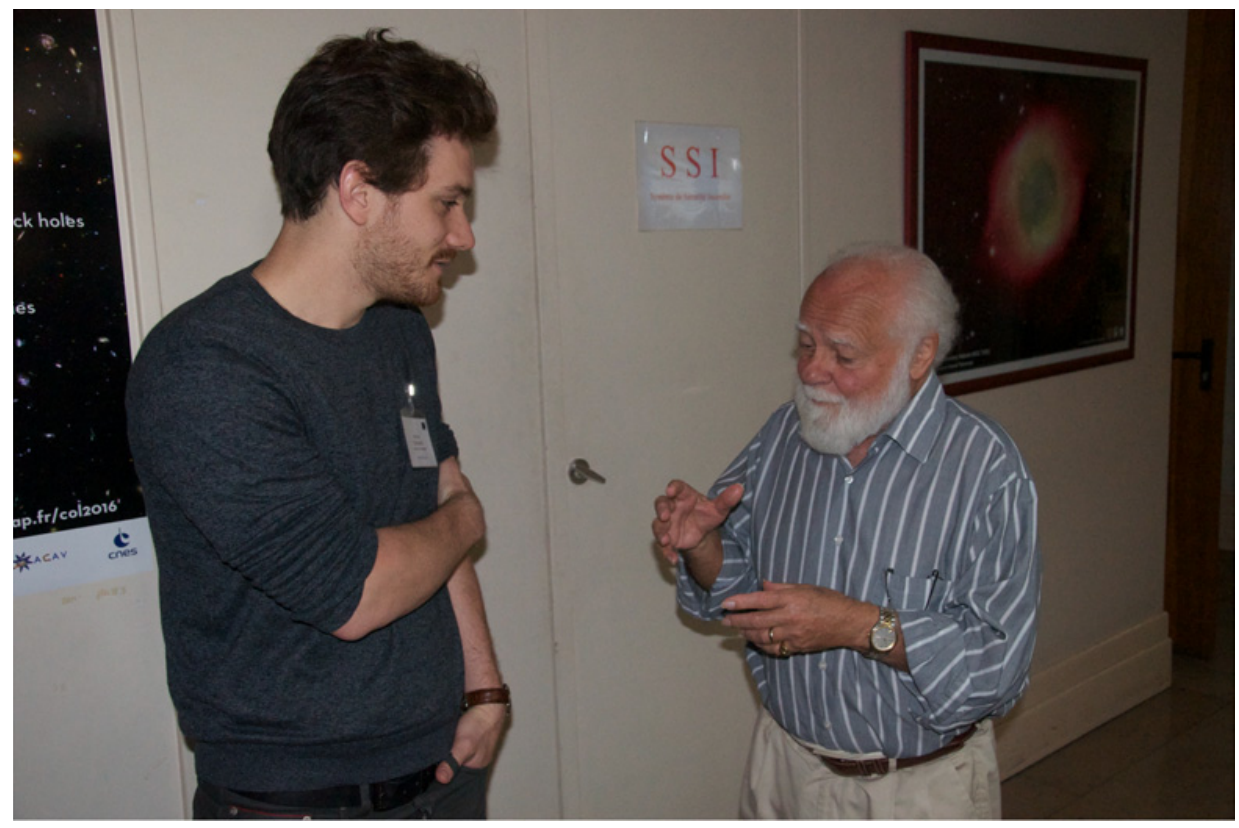

Antoine Strugarek and Douglas Gough

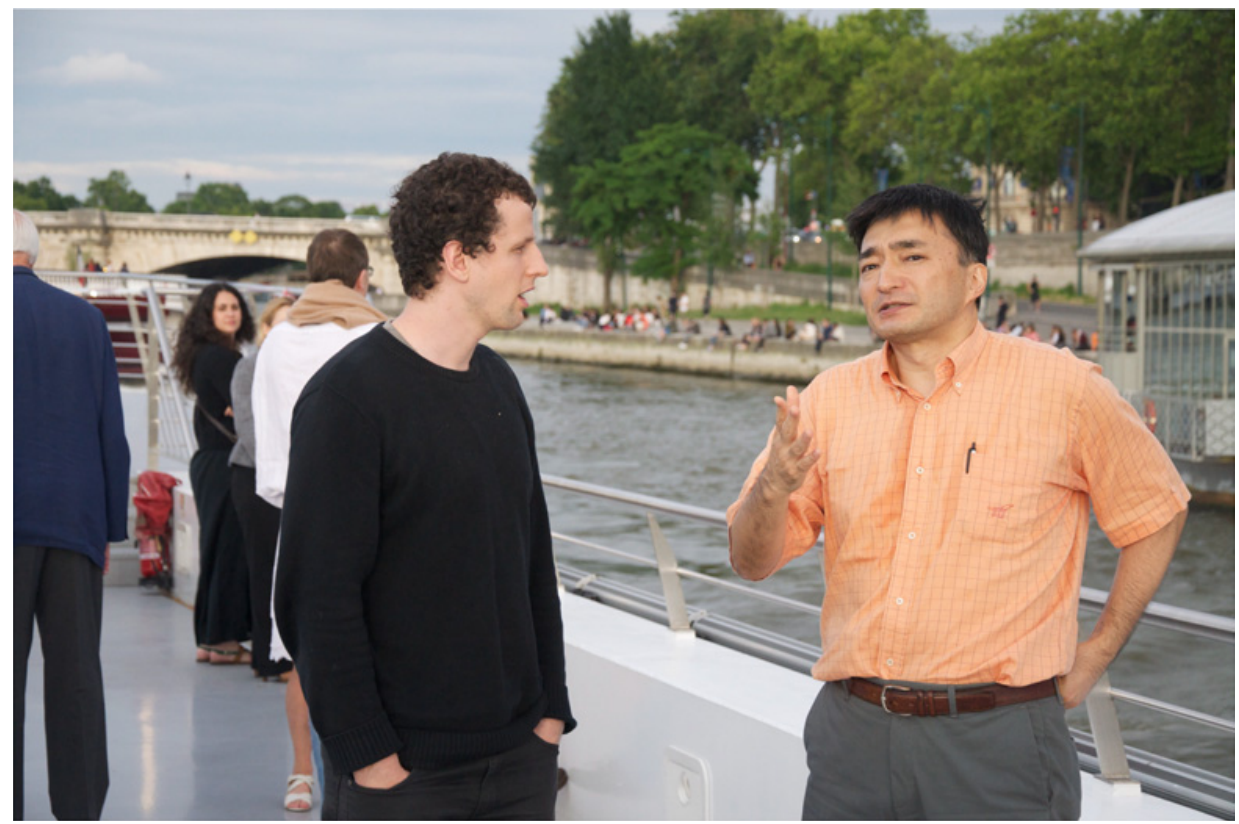

Antoine Riols-Fonclare and Yokoi Nobumitsu 


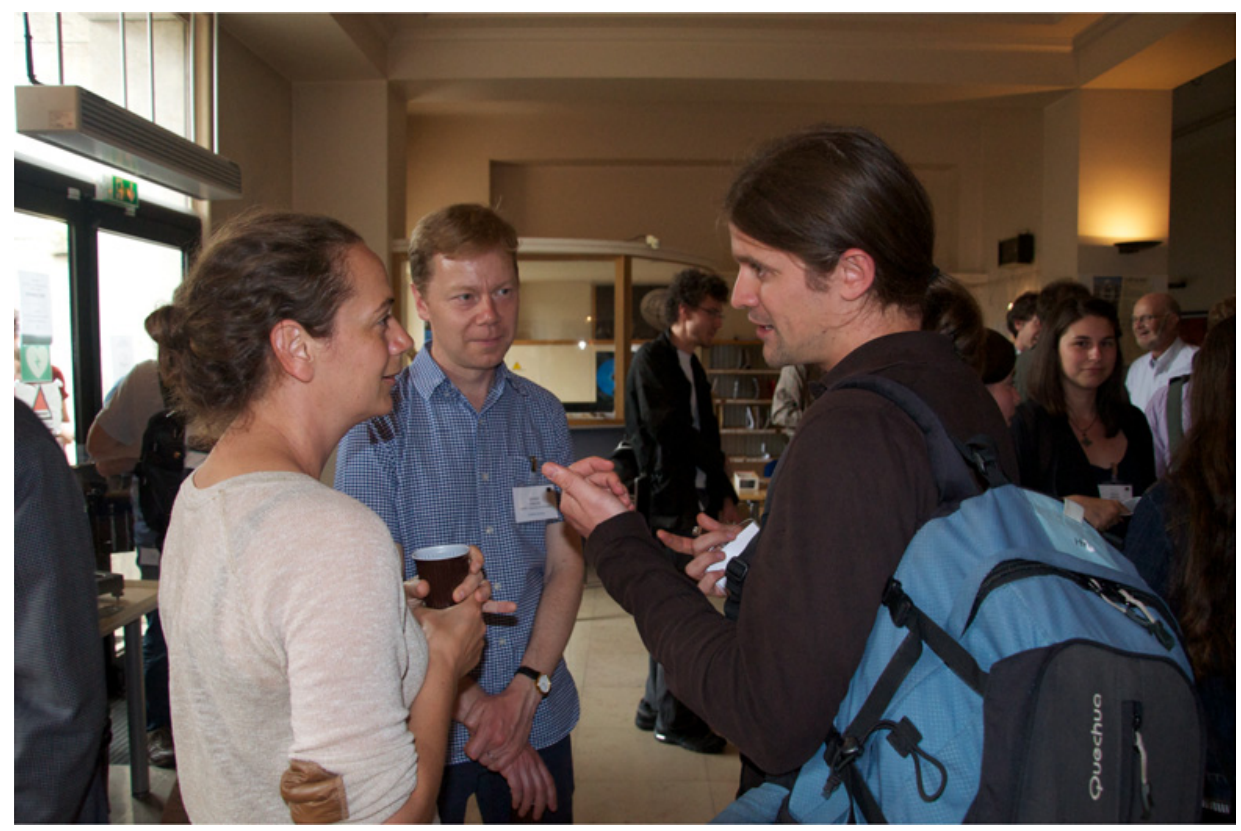

Laurène Jouve, Gordon Ogilvie and Benjamin Favier

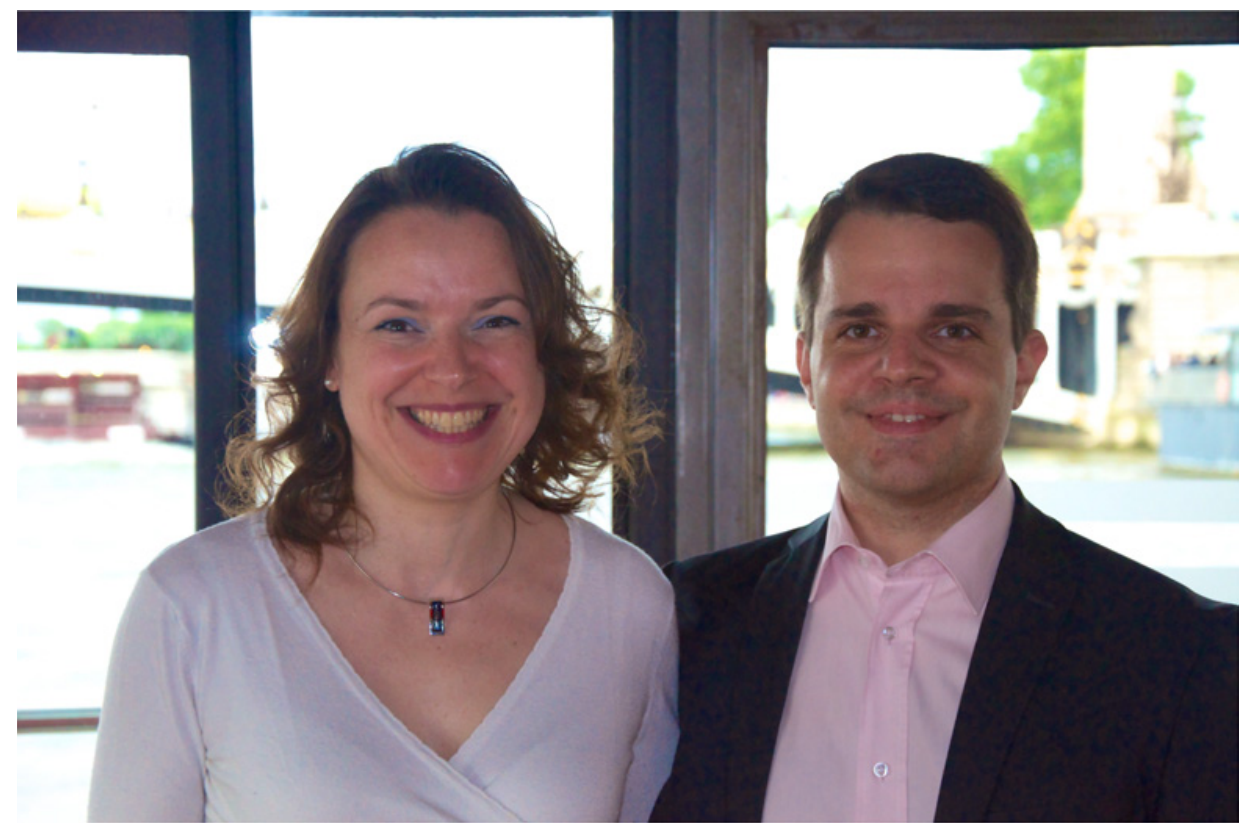

Coralie Neiner and Stéphane Mathis 